\title{
3 Entwicklung einer ethisch fundierten Unternehmensstrategie
}

\author{
Silke Bustamante
}

\subsection{Der strategische Managementprozess im Überblick}

Unternehmensstrategien betreffen Entscheidungen bzw. Maßnahmenkombinationen einer Unternehmung und relevanter Teilbereiche gegenüber ihrer Umwelt, welche die grundsätzliche Unternehmensentwicklung bestimmen. Ziel ist in der Regel die Sicherung der langfristigen Erfolgsposition des Unternehmens (Hungenberg 2004, S. 4). Die Integration des Verantwortungsgedankens in unternehmerisches Handeln sollte bei diesen grundsätzlichen Entscheidungen und Maßnahmenkombinationen ansetzen; sie betrifft die grundsätzliche Ausrichtung des Unternehmens. Die Entwicklung, Planung und Umsetzung der Strategien erfolgt im Rahmen eines zyklisch verlaufenden strategischen Managementprozesses, welcher - ebenso wie die Strategien - das Prinzip der Verantwortung berücksichtigen sollte und in das institutionelle und personelle Rahmengerüst des Unternehmens eingebettet ist (s. Abb. 11 und Kap. 2.5.2):

Ausgangspunkt des strategischen Prozesses bilden die übergeordneten Ziele bzw. das normative Grundgerüst des Unternehmens. Im Rahmen des strategischen Managementprozesses müssen Stakeholderinteressen identifiziert und bewertet, Ziele geklärt und in strategische Programme und Steuerungsroutinen integriert werden. Die im Rahmen des Strategieprozesses definierten Strategien und -programme haben ihrerseits Einfluss auf die Unternehmensstruktur sowie auf die Anforderungen an die Mitarbeiter und auf die erforderlichen Systeme. Wenngleich das normative Grundgerüst Teil des institutionellen Rahmens des Verantwortungsmanagements ist (s. Kap. 2.5.2), soll es aufgrund der Bedeutung für den strategischen Prozess und seinem engen Bezug zum strategischen Zielsystem in diesem Kapitel mit behandelt werden. In Anlehnung an Abbildung 11 lassen sich dann die folgenden Elemente des strategischen Managementprozesses zusammenfassen, welche zyklisch ineinandergreifen und in nachfolgenden Unterkapiteln weiter präzisiert werden (die Nummerierung der folgenden Abschnitte bezieht sich auf die Nummerierung in Abb. 11).

\section{1) Normatives Grundgerüst (s. auch Kap. 3.2)}

Das normative Grundgerüst setzt sich zusammen aus der Mission, den Unternehmenswerten, den Verhaltensgrundsätzen bzw. Leitlinien des Managements und der Vision. Die Mission detailliert den Unternehmenszweck bzw. die Daseinsberechtigung der Organisation, die Vision zeigt 


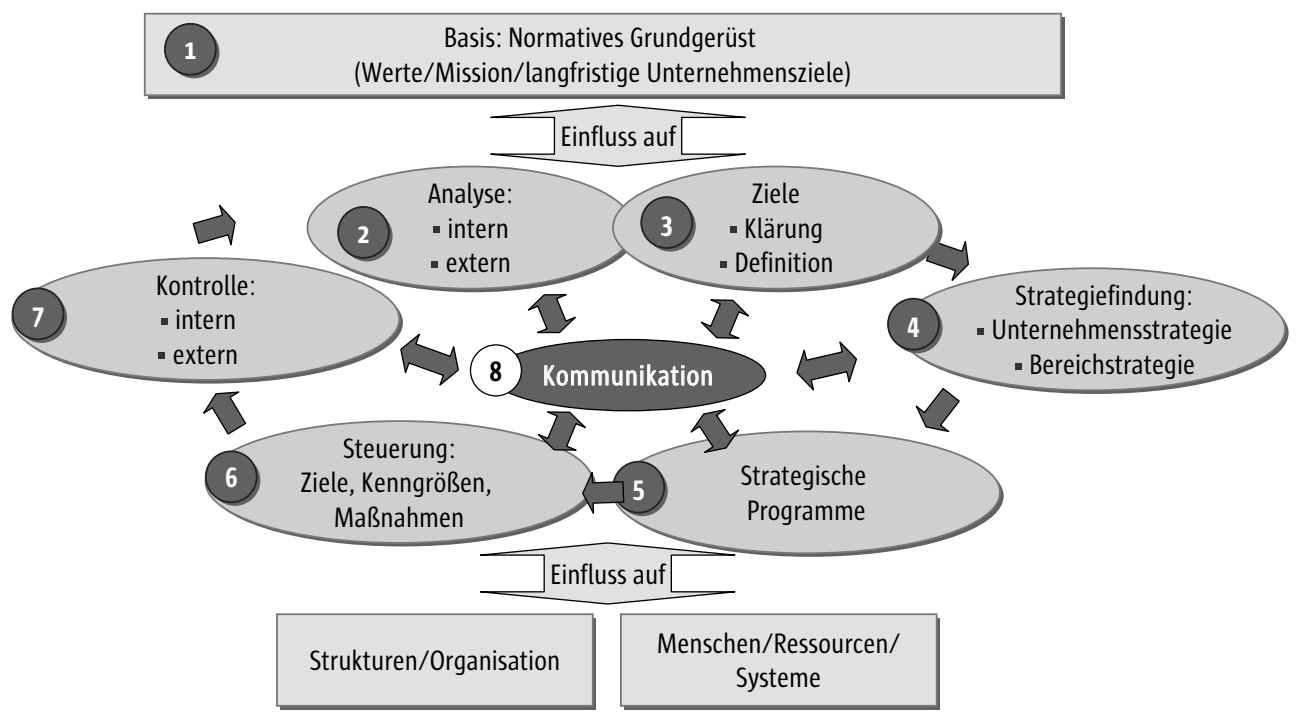

Abb. 11 Der strategische Managementprozess

auf, wohin sich das Unternehmen in den kommenden Jahren entwickeln möchte (Thompson/ Martin 2005 S. 42f.). Häufig werden Mission, Vision und Werte in Form eines Leitbildes formuliert. Es ist gestützt durch die Unternehmenskultur und die Unternehmensidentität (z.B. Meffert 2000, S. 71).

\section{2) Strategische Analyse (s. auch Kap. 3.3)}

Die strategische Analyse gibt Aufschluss über gesellschaftliche relevante und aus ethischer Sicht beachtenswerte Themen und Stakeholder sowie über den strategischen Handlungsspielraum und die Kernkompetenzen des Unternehmens. Im Rahmen der internen Analyse werden die Werte, Ressourcen, Kompetenzen und Prozesse des Unternehmens untersucht; die externe Analyse widmet sich gesellschaftlichen Trends und $\mathrm{He}-$ rausforderungen sowie den Interessen und Ansprüchen der wichtigsten Stakeholder. Resultat der Analyse ist die Konkretisierung von Themen, welche bei der Strategieentwicklung berücksichtigt werden sollten (z.B. Klimaproblematik, demographische Entwicklungen, Seuchen) und bei denen das Unternehmen aufgrund seiner Ressourcenposition besonderen Wert schaffen kann. Ausfluss der Analyse kann außerdem die Einsicht in die Notwendigkeit der Integration zusätzlicher Werte/Prinzipien in das kodifizierte Wertegerüst des Unternehmens sein, sodass dieses nachträglich angepasst wird (Münstermann 2007, S. 38 $)^{22}$.

\section{3)-5) Ziele, Strategien und strategische Programme (s. auch Kap. 3.4)}

Die strategische Analyse bildet die Basis zur Festlegung von Zielen und Strategien des Managements der Verantwortung. Es wird geklärt, welchen Einfluss die Übernahme von Verantwortung auf andere Unternehmensziele wie Kundenzufriedenheit, Kosten oder Qualität hat (Münstermann 2007, S. 36f.). Außerdem werden Inhalte und Schwerpunkte, Adressaten und Formen des Verantwortungsmanagements für das gesamte Unternehmen bzw. einzelne Geschäftsbereiche festgelegt (Münstermann 2007, S. 47; Göbel 2006, S. 148-150). Strategische Programme konkretisieren Strategien für die betrieblichen Funktionen und Unternehmensbereiche über die Zeit auf die Gegenwart; sie gelten auch als Bindeglied zur operativen Planung. Strategische Ziele und Wege der Zielerreichung sind dabei so eng miteinander verzahnt, dass eine getrennte Behandlung der Themenblöcke hier als nicht sinnvoll erachtet wird.

22 Münstermann (2007, S. 38) spricht von einem Einfluss der Unternehmensziele auf das Wertegerüst des Unternehmens, nichtsdestotrotz ist die Analyse der wesentliche Treiber sowohl für die Unternehmensziele und damit auch gleichzeitig für das Normsystem. 


\section{6)-7) Steuerung und Kontrolle (s. auch Kap. 3.5)}

Wesentlich für die Umsetzung der Strategie sind interne Steuerungskonzepte, welche die Ableitung von mittel- und kurzfristigen Zielen, die Definition von Maßnahmen und Kenngrößen (sogenannte Key Performance Indikatoren) für einzelne Unternehmensbereiche und Funktionen beinhalten.

Die Kontrolle dient dem Soll-Ist Abgleich der definierten Kenngrößen. Ihre Ergebnisse bilden den Ausgangspunkt eines neuen Strategieprozesses und können zur Umsteuerung der Strategie führen. Kontrolle kann sowohl intern als auch extern realisiert werden (externen Audits oder Ratings); sie ist dann Bestandteil des Managementprozesses, wenn sie intentional durch das Management herbeigeführt wird. Steuerungsund Kontrollkonzepte werden häufig als ein den gesamten Managementprozess begleitendes Instrument gesehen, da die Steuerung und Kontrolle bereits bei den strategischen Prämissen ansetzen sollte. Da die Ergebnisse der Kontrolle den (nächsten) strategischen Planungsprozess maßgeblich beeinflussen, wird die Kontrolle hier als Bindeglied zur Analyse gesehen.

\section{8) Kommunikation (s. auch Kap. 3.6)}

Die Kommunikation ist einerseits integraler Bestandteil des strategischen und operativen Managements bzw. der internen Steuerung (Zerfaß 2004 S. 29off.); andererseits dient sie der Positionierung des Unternehmens bei seinen relevanten Stakeholdern und ist damit Bestandteil des externen Marketings ${ }^{23}$. Im Zuge der internen Steuerung geht es darum, die Ziele, geplanten Projekte und Maßnahmen und deren Ergebnisse an die Mitarbeiter des Unternehmens sowie ggf. am Prozess beteiligte externe Stakeholder zu kommunizieren, sich Feedback einzuholen, um Umsteuerungsmaßnahmen ableiten zu können (Brandes; Schabel; Wache 2005, S. 59). Gleichzeitig beeinflusst die interne Kommunikation die Unternehmenskultur (Deekeling; Fiebig 1999, S. 241), durch welche das Prinzip der Verantwortung wesentlich getragen wird, und wirkt auf das Selbstbild des Unternehmens (Münstermann 2007, S. 171). Im Zuge der externen Kommunikation geht es darum, die Wahrnehmung des Verantwortungsmanagement durch externe Stakeholder zu gestal-

23 Zur Abgrenzung zwischen externen und internen Marketing vgl. Schulz 1994, S. $531 \mathrm{ff}$. ten und so das Fremdbild dem angestrebten Bild in der Öffentlichkeit anzunähern (Münstermann 2007, S. 101), aber auch darum, die Stakeholder durch Stakeholderdialoge und Ergebniskommunikation in den strategischen Prozess einzubeziehen. Letzteres ergibt sich nicht zuletzt aus dem Verständnis des Verantwortungsmanagements als Management eines Interessenausgleichs zwischen unterschiedlichen Stakeholdergruppen.

\subsection{Die Integration des Verantwortungsprinzips in das normative Grundgerüst des Unternehmens}

Die Integration des Gedankens der Verantwortung in den strategischen Managementprozess beginnt mit dessen Verankerung in der Mission, den Werten und Leitlinien sowie den langfristigen Unternehmenszielen. Ziele werden dabei verstanden als „Aussagen oder Vorstellungen über angestrebte Zustände, die durch Handlungen hergestellt werden sollen“ (Kubicek 1981, S. 458). Solche Aussagen werden sowohl bei der Formulierung der Mission (z.B. Mission von Helios: „Wir wollen Qualität in der Medizin für jeden erfahrbar machen “24), der Werte und der langfristigen Unternehmensziele (z.B. Steigerung von Patientennutzen und „Qualitätsführerschaft") als auch auf der strategischen und operativen Managementebene getroffen (Meffert 2000, S. 71). Die Verortung des Zielbildungsprozesses im Managementprozess ist daher nicht eindeutig festlegbar. Während übergeordnete Ziele als Leitlinien bzw. Prämissen den Prozess der Bildung und Auswahl von Strategien bestimmen (bzw. darüber hinaus auch Eingang in den Analyseprozess finden), können konkrete Handlungsziele in der Regel erst im Anschluss an die Strategiebestimmung formuliert werden (Schreyögg 1984, S. 87).

Die Formulierung von Mission, Vision und Werten ist wesentlich bestimmt von den Motiven und Wertvorstellungen der Führungskräfte sowie von den Anforderungen des externen Umfeldes.

Auf Motive wurde im Rahmen der ethischen und strategischen Begründungsansätze in Kapitel 2 bereits kurz eingegangen. Einen Überblick

24 S. Unternehmensseite von Helios: http://www.helioskliniken.de/ueber-helios/unternehmensportrait.html; abgerufen am 15.1.2010. 


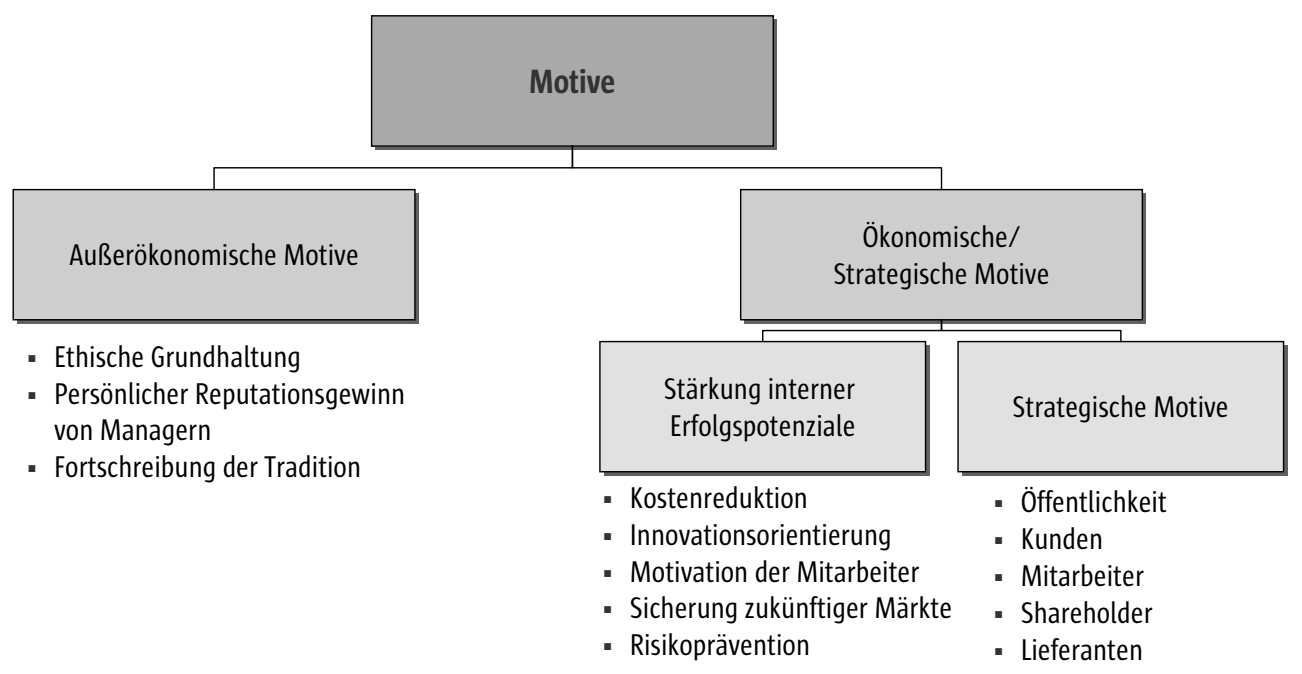

Abb. 12 Motive des Verantwortungsmanagements

über die Motive des Verantwortungsmanagement gibt Abbildung 12.

Sowohl in die Mission als auch in Vision und Unternehmenswerte können nun Aspekte der Verantwortung integriert werden. Novartis definiert seine Mission zum Beispiel wie folgt: „Leben zu verlängern, Leiden zu lindern und die Lebensqualität von Menschen nachhaltig zu verbessern." Daraus ergibt sich der Auftrag zu Innovation und Forschung sowie zur Übernahme von gesellschaftlicher und sozialer Verantwortung (Novartis 2010). Henkel (2010) integriert Kundenorientierung, Nachhaltigkeit und gesellschaftlich verantwortliches Handeln in seine Werte (s. Abb. 13).

Häufig verpflichten sich Unternehmen außerdem zur freiwilligen Einhaltung von Verhaltenskodizes, welche in Form von Prinzipien oder Standards das (angestrebte) Geschäftsgebaren im Markt beschreiben. Sie werden von Unternehmen, Verbänden oder anderen Institutionen verfasst und beziehen sich in der Regel auf Arbeitsbedingungen, Umwelt- oder Verbraucherschutz. Einer der frühesten unternehmensübergreifenden Initiativen in diesem Zusammenhang ist das im Jahr 1973 auf dem European Management Forum abgefasste Davoser Manifest, welches als Grundlage der Erstellung von Führungsgrundsätzen im Sinne der Führungsethik dient. In dem Manifest werden Ziele wie zum Beispiel der Dienst am Kunden, am Mitarbeiter, das Ziel der sozialen Verantwortung für die Gesellschaft, der Interessenausgleich zwischen Arbeitgebern und -nehmern sowie Qualitätsansprüche an Produkte und Produktionsprozesse aufgestellt. $\mathrm{Zu}$ den gegenwärtig bekanntesten und weltweit anerkannten Kodizes zählt der UN Global Compact, ein 1999 vom damaligen UN Generalsekretär Kofi Annan initiierter „Pakt“, welcher Unternehmen aufruft, sich zur Einhaltung von 10 grundlegenden Prinzipien verantwortungsvollen Handelns zu verpflichten (s. Abb. 14).

Unternehmensspezifische Verhaltenskodizes (oder "Codes of Conduct") enthalten allgemeine Unternehmens- und Handlungsgrundsätze und sollen Führungskräfte und Mitarbeiter unterstützen, ethische und rechtliche Fragestellungen angemessen zu beantworten. Beispielsweise beziehen sich die Geschäftsgrundsätze von SAP (SAP 2006) auf Rechtstreue, Loyalität, Korruption, Vertraulichkeit, Finanzwesen, aber auch das Verhalten gegenüber den wichtigsten Stakeholdergruppen.

Der im November 2010 verabschiedete Standard ISO 26000 könnte dabei zukünftig unter anderem als Grundlage für die Entwicklung von Leitsätzen und Normen im Unternehmen dienen (International Organization for Standardization (ISO), 2010). Er beschreibt, was unter verantwortungsvollem Management international verstanden wird und soll darüber hinaus anhand von Beispielen zeigen, wie sich diese Leitsätze im operativen Geschäft von Organisationen niederschlagen können.

Im Zuge der Analyse von Stakeholderanliegen (s. Kap. 3.3.2) kommt es häufig zur Identifikation 


\section{Unsere Werte}

Wir stellen unsere Kunden in den Mittelpunkt unseres Handelns.

Wir denken voraus und gehen auf die Wünsche und Bedürfnisse unserer Kunden ein, indem wir höchsten Kundennutzen, ausgezeichnete Qualität sowie innovative Marken und Technologien bieten.

\section{Wir schätzen, fordern und fördern unsere Mitarbeiter.}

Wir begegnen uns mit Respekt und Achtung, vertrauen einander und entwickeln unsere Fähigkeiten gemeinsam weiter. Wir erwarten von jedem Einzelnen eigenverantwortliches Handeln und ein hohes Leistungsniveau, um unseren Erfolg als Unternehmen zu sichern. Wir streben herausragenden und nachhaltigen Erfolg an.

Wir fühlen uns als leistungsorientiertes Unternehmen verpflichtet, den Wert unseres Unternehmens zu steigern und unseren Aktionären eine attraktive Rendite zu sichern. Wir verpflichten uns, unsere führende Rolle im Bereich Nachhaltigkeit auszubauen. Wir bieten Produkte, Technologien und Prozesse, die höchste Standards erfüllen. Wir verpflichten uns, für die Sicherheit und Gesundheit unserer Mitarbeiter, den Schutz unserer Umwelt und die Lebensqualität der Menschen in unserem Umfeld zu sorgen.

Wir gestalten unsere Zukunft auf dem Fundament eines erfolgreichen Familienunternehmens.

Basierend auf unseren Werten und unserer langjährigen erfolgreichen Geschichte verfolgen wir eine langfristig ausgerichtete Unternehmensentwicklung, die durch faires unternehmerisches Handeln und eine solide Finanzierung geprägt ist.

Abb. 13 Werte bei Henkel (Henkel 2010)

\section{Human Rights}

Principle 1: Businesses should support and respect the protection of internationally proclaimed human rights; and

Principle 2: make sure that they are not complicit in human rights abuses.

\section{Labour Standards}

Principle 3: Businesses should uphold the freedom of association and the effective recognition of the right to collective bargaining;

Principle 4: the elimination of all forms offorced and compulsory labour;

Principle 5: the effective abolition of child labour; and

Principle 6: the elimination of discri mination in respect of employment and occupation.

\section{Environment}

Principle 7: Businesses should support a precautionary approach to environmental challenges;

Principle 8: undertake initiatives to promote greater environmental responsibility; and

Principle 9: encourage the development and diffusion of environmentally friendly technologies

\section{Anti-Corruption}

Principle 10: Businesses should work against all forms of corruption, including extortion and bribery.

Abb. 14 Die 10 Prinzipien des United Nations Global Compact (www.unglobalcompact.org) 
von Interessenkonflikten zwischen einzelnen Stakeholdergruppen. Bei der Lösung von Konflikten legitimer Stakeholderanliegen können sogenannte ethische Vorzugsregeln angewandt werden. Als Beispiel seien an dieser Stelle die Vorzugsregeln bzw. Prinzipien der „Übelabwägung“ (Korff 1979, S. 82) genannt:

- Unter sonst gleichen Umständen ist eine Handlungsweise, die ein bestimmtes Übel wahrscheinlich zur Folge hat, einer anderen Handlungsweise vorzuziehen, die dieses mit Sicherheit zur Folge hat.

- Unter sonst gleichen Umständen ist bei Übeln, die unvermeidlich sind, das Geringere dem Größeren und das kürzer Dauernde dem länger Dauernden vorzuziehen.

- Unter sonst gleichen Umständen ist im Konfliktfall zugunsten der Vielen und nicht der Wenigen zu entscheiden.

- Bei Tatsachenzweifel und trotzdem gegebener Notwendigkeit zur Entscheidung ist einer reversiblen vor einer irreversiblen Entscheidung der Vorzug gegeben.

\subsection{Die strategische Analyse}

Die strategische Analyse schafft die informatorischen Voraussetzungen für die Ableitung strategischer Optionen und deren Bewertung und besteht aus der Analyse des eigenen Unternehmens (interne Analyse) einerseits und der Analyse des Umfelds (externe Analyse) andererseits (Schreyögg; Koch 2007, S. 75). Die interne Analyse gibt Auskunft über Kernkompetenzen und Ressourcen des Unternehmens (Thompson; Martin 2005, S. 21off.), Stärken und Schwächen gegenüber Konkurrenten, Wertvorstellungen der Unternehmensmitglieder und Schnittstellen des Unternehmens zum gesellschaftlichen Gesamtsystem. Sie spielt eine wesentliche Rolle für die Beurteilung externer Anforderungen und bestimmt den Spielraum, auf Chancen und Risiken adäquat antworten zu können (Schreyögg; Koch 2007, S. 75). Die Analyse des externen Umfeldes hingegen dient der Identifizierung dieser Chancen und Risiken, insbesondere derjenigen, die im Zusammenhang mit Ansprüchen und Anforderungen relevanter Stakeholder stehen (Münstermann 2007, S. 83).

\subsubsection{Die interne Analyse}

Zu den Inhalten der internen Analyse gehören

- die Beschreibung und Beurteilung der Ressourcen und Fähigkeiten (Schreyögg; Koch 2007, S. 88ff.),

- die Identifikation ökologischer und sozialer Ansatzpunkte im Wertschöpfungsprozess (Porter; Kramer 2006, S. 5) und

- die Analyse und Interpretation der Wertvorstellungen der Unternehmensmitglieder.

Während die Beschreibung und Beurteilung der Ressourcen und Fähigkeiten Aufschluss gibt, ob die Organisation über die Übernahme von Verantwortung für bestimmte Themen Wert schaffen bzw. sich differenzieren kann, werden durch die Analyse der Wertschöpfungskette potenzielle Schnittstellen zu externen Stakeholdern aufgedeckt und für die Stakeholder- und Issue-Analyse wesentliche Themen identifiziert. Die Analyse und Interpretation der Wertvorstellungen sind insofern relevant, als dass sie das normative Gerüst eines Unternehmens mit formen (s. Kap. 3.2) und die Beurteilung relevanter Stakeholder und Themen sowie strategischer Optionen beeinflussen.

\section{Die Analyse der Ressourcenposition}

Zu den strategischen Ressourcen eines Unternehmen werden nach Hofer/Schendel (1978) finanzielle, physische (Gebäude, Ausrüstung u.ä.), personelle, organisatorische (Informationssysteme, Integrationsabteilungen) und technologische Ressourcen (Qualitätsstandards, Marken-Know-how, Forschungs-Know-how etc.) gezählt. Darüber hinaus werden häufig noch übergreifende Fähigkeiten als strategisch besonders relevant beschrieben. Nach Prahalad/Hamel (1990) sind dies z.B. unternehmenskulturell verankerte Prozesse, die den Leistungsvollzug im Unternehmen auf meist unsichtbare Weise mitstrukturieren (Schreyögg; Koch 2007, S. 94). Diese Fähigkeiten werden auch als Kernkompetenzen bezeichnet.

Im Zuge eines Managements sozialer Verantwortung ist insbesondere von Interesse, inwieweit organisatorische und personelle Ressourcen Strategien der Verantwortung unterstützen und ermöglichen. Zusätzlich stellt sich die Frage, inwieweit spezifisches Know-how in ausgewählten Bereichen die Übernahme von Verantwortung für 
bestimmte Themen besonders sinnvoll macht (z.B. Einsatz des Kommunikations-Know-hows von Telekommunikationsunternehmen zur Förderung interkultureller Kommunikation). Eine Beurteilung der Ressourcen und Fähigkeiten mit Blick auf diese Fragestellungen sollte durch eine vergleichende Beurteilung der Ressourcenposition wesentlicher Wettbewerber unterstützt werden (Thompson; Martin 2006, S. 212). Diese erlaubt, eigene Stärken und Schwächen zu identifizieren (Schreyögg; Koch 2007, S. 89) und gegebenenfalls Ideen und Ansatzpunkte für ein erfolgreiches Verantwortungsmanagement zu generieren.

\section{Die Analyse der Wertschöpfungskette}

Die Wertschöpfungskette beschreibt die prozessuale Abfolge bzw. die Zusammenhänge zwischen allen Aktivitäten, welche ein Unternehmen ausführt, um ihr Produkt bzw. ihre Leistung entwerfen, produzieren, vertreiben, ausliefern und unterstützen zu können (Porter 1980). Diese Aktivitäten werden gemäß ihres Wertschöpfungsbeitrages von Beginn des Leistungsprozesses (z.B. Einkauf der Materialien) bis zum Leistungsergebnis prozessual angeordnet.

Die Wertschöpfungskette gibt u.a. Aufschluss über mögliche Schnittstellen des Unternehmens zur externen Umwelt und dient so als Instrument, Issues und Stakeholder zu identifizieren (Porter; Kramer 2006). Insbesondere bei Integration vorund nachgelagerter Aktivitäten (z.B. Produktion der Medizintechnikgeräte, Entsorgung von Geräten) in eine unternehmensübergreifende Wertkette ergeben sich wichtige Hinweise auf gesellschaftliche Auswirkungen des Wertschöpfungsprozesses; allerdings stellt sich in diesem Zusammenhang dann auch schnell die Frage nach den Grenzen der Verantwortung.

\section{Die Analyse und Interpretation der Wertvorstellungen der Unternehmensmitglieder}

Die Analyse der kulturellen Maßstäbe und Ideale von Mitarbeitern und Unternehmensleitung, welche sich im gegenwärtigen Zustand der Unternehmensidentität widerspiegeln (Meffert 20oo, S. 70), ist für das Management der sozialen Verantwortung aus drei Gründen von Bedeutung:

- Erstens gibt sie Auskunft über aus Sicht des Managements erstrebenswerte strategische Ziele
(Meffert 2000, S. 70). Zusammen mit der externen Analyse (Erwartungen von Stakeholdern und gesetzliche Vorschriften) sollte diese Ergebnisse Einflussgröße bei der Formulierung von Werten und Verhaltenskodizes sein (Schaumann 2006).

- Zweitens weist sie auf Handlungsbedarf hin, sofern von außen an das Unternehmen herangetragene Werterwartungen nicht mit den Vorstellungen der Unternehmensmitglieder übereinstimmen (Münstermann 2007, S. 69ff.).

- Drittens sind sie Maßstab für Veränderungsprozesse, bei welchen analysiert werden muss, welches Maß an Veränderung ein Unternehmen verträgt, ohne dass es sich untreu wird (Meffert 2000, S. 71).

\subsubsection{Die externe Analyse}

Bei der Analyse des Unternehmensumfeldes unterscheidet man im Regelfall die Analyse der globalen Umwelt (politische, natürliche, technologische, rechtliche, makroökonomische und sozio-kulturelle Einflussfaktoren) von der des unmittelbaren Wettbewerbsumfeldes (potenzielle und aktuelle Wettbewerber, Lieferanten, Nachfrager, Politik, Substitutionsprodukte). Während die Analyse der globalen Umwelt potenziell relevante Trends und Entwicklungen identifizieren soll, geht es bei der Analyse des Wettbewerbsumfeldes um die zentralen Einflusskräfte auf die Branche und das Unternehmen (Schreyögg; Koch 2007, S. $77 f f$.). Die externe Analyse ist dabei auf den spezifischen Erkenntnisgegenstand zuzuschneiden; der für das Ziel der Analyse relevante Umweltausschnitt muss klar definiert sein (Schreyögg; Koch 2007, S. 77).

Im Zuge des Managements sozialer Verantwortung geht es im Wesentlichen um einen Ausgleich der Interessen unterschiedlicher relevanter Stakeholdergruppen und die Analyse solcher Themen und Trends, welche in Zukunft zu potenziellen Konfliktsituationen führen könnten. Aus diesem Grund erfolgt im Folgenden eine Konzentration auf die Aspekte der Analyse, welche als Crundlage zur Ableitung von konsensfähigen und verantwortlichen Strategien dienen können:

- Die Stakeholderanalyse ermöglicht die Identifikation der Schnittstellen des Unternehmens zu relevanten gesellschaftlichen Cruppierungen und die Analyse der Erwartungen dieser Gruppierungen an das Unternehmen. 
- Die allgemeine Analyse sozialer Themen (sogenannte „Issues“) und globaler sozialer, umweltbezogener und rechtlicher Trends gibt Hinweise auf möglichste Erwartungen von Stakeholdern in der Zukunft sowie potenzielle zukünftige Konfliktsituationen.

Die Bewertung und Beurteilung von Stakeholdern und Issues ist Grundlage der Priorisierung im Falle von Ressourcen- oder Interessenkonflikten.

\section{Die Stakeholderanalyse}

Im Rahmen der Stakeholderanalyse geht es darum, potenziell relevante Stakeholder wahrzunehmen und ihre Anliegen, Ziele, Motive, Stärke, Verhaltensweisen u.ä. zu erkennen und zu analysieren.

Wie in Kapitel 2.2.2 ausgeführt, sind potenzielle Stakeholder eines Unternehmens diejenigen Personen oder Personengruppen, die sich von der Unternehmenstätigkeit in irgendeiner Art und Weise direkt oder indirekt betroffen fühlen und/oder Einfluss auf das Unternehmen ausüben können (Rhenman 1968, S. 25). Es geht also in der Regel um eine wechselseitige Interaktion oder Einflussnahme zwischen dem Unternehmen und seinen Stakeholdern (Münstermann 2007, S. 84).

Offensichtlich als Stakeholder erkennbar sind dabei jene Personen/Gruppen zu denen das Unternehmen vertragliche Beziehungen unterhält, das heißt Mitarbeiter ${ }^{25}$, Kunden, Lieferanten, Fremdkapitalgeber; in Krankenhäusern außerdem Sanitätshäuser, Pharmaunternehmen, Pflegedienste, öffentliche Träger u.ä. (Göbel 2006, S. 117) ${ }^{26}$. Auch Personen oder Institutionen, welche die Interessen der vertraglichen Partner stellvertretend wahrnehmen, wie z.B. Personalvertretungen, Verbände, Gewerkschaften, Verbraucherschutzvereinigen u.ä. müssen demnach zu den Stakeholdern gezählt werden. Der Staat als Empfänger von Steuern und Financier von Infrastruktur steht - wenn auch nicht in einer vertraglichen Beziehung - ebenso in einem klaren wechselseitigen Austauschverhältnis mit dem Unternehmen; in der Gesundheitsbranche ist er teilweise selbst

25 Zur Doppelrolle von Mitarbeitern und angestellten Führungskräften vgl. Kapitel 2.2.2.

26 Zur Einordnung von Führungskräften und Eigentümern vgl. Kapitel 2.2.
Eigentümer von Krankenhäusern und beeinflusst das Management der Krankenhäuser wesentlich über Regulierungen und Gesetze. Zusätzlich werden in der Regel die Öffentlichkeit, die Medien, Nichtregierungsorganisationen etc. zu möglichen Stakeholdern gezählt (Göbel 2006, S. 117).

Die Relevanz von Stakeholdern ist abhängig von der Branche, dem Unternehmen, der spezifischen Unternehmenssituation bzw. dem Unternehmensbereich, dem aktuellen Rechtsrahmen und dem Zeitpunkt der Analyse; so sind $\mathrm{zu}$ verschiedenen Zeitpunkten unterschiedliche Themen relevant, können unterschiedliche $\mathrm{Er}$ wartungen bestehen und unterschiedliche Gesetze gelten (Göbel 2006, S. 122ff.). Daher sollte die Wahrnehmung und Identifikation möglicher Stakeholder durch Social Issue Analysen (Rückschlüsse aus gesellschaftlich relevanten Themen auf betroffene Stakeholder), Wertkettenanalysen und Stakeholderdialoge unterstützt werden (s. nachfolgende Abschnitte).

Um die identifizierten Stakeholdergruppen zu analysieren, eignen sich grundsätzlich die gängigen Methoden der Marktforschung (Umfragen Experteninterviews, Auswertung von Massenmedien etc.), insbesondere aber der direkte Dialog mit den Stakeholdern und die Gestaltung von Möglichkeiten des Feedbacks durch betroffene Stakeholder. Cestaltungsformen des Stakeholderdialoges sind z.B. Stakeholderforen bzw. Multistakeholderinitiativen, Gremien, Fachworkshops, Messen, Podiumsdiskussionen, Fokusgruppen, Internetblogs und Vorträge.

\section{Social Issue Analyse}

Als Social Issues werden Themen öffentlichen Interesses mit wahrscheinlichem Konfliktpotenzial und potenziellen Einfluss auf das Unternehmen, welche in der Regel über Interessengruppen an das Unternehmen herangetragen werden bezeichnet (Röttger 2001, S. 19). Freeman bezeichnet die Issue Analyse als einen von drei Schritten einer strategischen Stakeholderanalyse (Freeman 1984, S. 92):

1. Wer sind die Stakeholder und wie werden diese in politischer, wirtschaftlicher und sozialer Sicht beeinflusst?

2. Welche Werte haben Organisationsmitglieder und Schlüssel-Stakeholder?

3. Welches sind die wesentlichen politischen, technologischen und wirtschaftlichen Issues 
der nächsten to Jahre und wie beeinflussen sie das Unternehmen und die Stakeholder?

Die Social Issue Analyse verfolgt zum einen das Ziel, die Anliegen identifizierter Stakeholder zu untermauern und zu präzisieren und bisher nicht identifizierte Stakeholder wahrzunehmen (s.o.). Durch das Zusammenführen von Inhalten/Themen (z.B. Umweltverschmutzung, $\mathrm{CO}_{2}$-Ausstoß) und Stakeholdern mit ihren Ansprüchen (z.B. Forderungen der Kunden/von NGO's) können Themen gebündelt und ihr Einfluss auf unterschiedliche Stakeholdergruppen aufgezeigt werden (vgl. Abb. 15).

Zum anderen dient die Social Issue Analyse der Prognose von zukünftig relevanten gesellschaftlichen Themen und deren Auswirkung auf heutige und zukünftige Stakeholder. Es geht also darum, frühzeitig über sogenannte „weak signals“ (Ansoff 1980), die für die nächsten Dekaden wesentlichen Issues zu identifizieren und ihren Einfluss auf das Unternehmen und seine Stakeholder zu erkennen, und so der Organisation mehr Handlungsmöglichkeiten und Einflussmöglichkeiten zu geben.

Die Issue Identifikation kann sich an dem Analyseraster der PESTLE Analyse orientieren, bei der Einflüsse des politischen, wirtschaftlichen (economic), sozialen und technologischen, rechtlichem (legal) und natürlichem (ecological) Umfeldes auf das Unternehmen bzw. seine wesentlichen Stakeholder analysiert werden. Je nach Gerichtetheit der Suche unterscheidet man zwischen dem Issue Scanning und dem Issue Monitoring. Das Scanning ist die eher intuitive und ungerichtete Beobachtung des Unternehmensumfeldes und dient dazu, Zusammenhänge oder Muster zu erkennen, die ggf. relevant werden. Wichtigste Instrumente des Scanning sind die Analyse von Massenmedien, Internet, Unternehmensmitteilungen und sonstigen Veröffentlichungen. Das Monitoring als gerichtete Suche und Verfolgung von Issues nutzt zusätzliche Befragungen und Expertengespräche.
Aus einer Fülle identifizierter Issues und Themen sind jene herauszufiltern, welche das Unternehmen als wesentlich für sich erachtet. Dazu werden zunächst Issues gebündelt und ggf. zu Trends zusammengefasst. Eine Priorisierung der Issues sollte in Verbindung mit der Bewertung der betroffenen Stakeholder durchgeführt werden, da durch diese die Themen an das Unternehmen herangetragen werden (vgl. Abb. 15).

\section{Die Auswahl und Bewertung relevanter Stakeholder und Issues}

Abschließender Schritt der externen Analyse ist die Bewertung bzw. Priorisierung der Stakeholder und der Ansprüche und Issues, für welche sich das Unternehmen verantwortlich erklärt. Die Bewertung und Priorisierung ist aus zwei Gründen erforderlich: Zunächst geht es darum, aus einer Fülle von Stakeholdern, Anliegen und Issues jene herauszufiltern, mit denen sich die Organisation im Zuge eines Managements der sozialen Verantwortung beschäftigen kann und sollte, das heißt für die sie sich verantwortlich erklärt. Zusätzlich dient eine Bewertung als Grundlage, unterschiedliche Anliegen und Interessen gegeneinander abzuwägen und durch die Formulierung verantwortlicher Strategien eine konsensfähige Lösung zu finden (s. Kap. 3.3.2).

Diese Bewertung kann sowohl mittels ethischer Maßstäbe als auch aus strategischer Sicht (welche Ansprüche sind mit Blick auf die Bedeutung der Stakeholder und deren Anliegen für das eigene Unternehmen sinnvollerweise zu erfüllen) erfolgen (Müller/Hübscher 2007, S. 145). Wie in Kapitel 2.2.2 diskutiert, findet eine ethische Bewertung seine Grundlage im Wertegerüst des Unternehmens und beschäftigt sich mit der Frage der Legitimität der Stakeholderanliegen bzw. der Betroffenheit der Stakeholder. Es geht darum, zu entscheiden, inwieweit das Unternehmen mit Blick auf seine Wertvorstellungen und gesellschaftliche Erwartungen sich zur Erfüllung

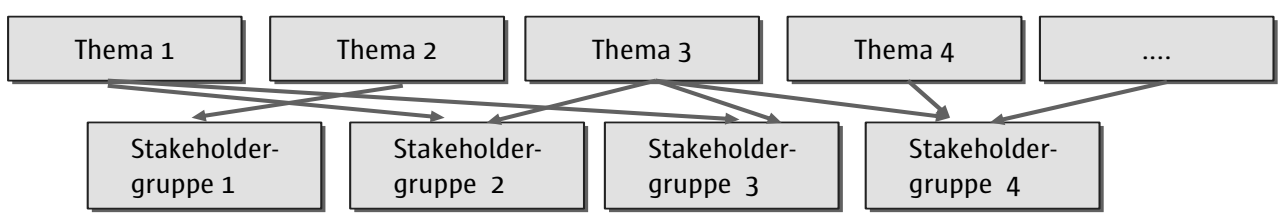

Abb. 15 Identifikation von Stakeholdern über Social Issues 
der Stakeholderanliegen verpflichtet sieht. Aus strategischer Sicht ist außerdem der Einfluss und das Ressourcen- und Machtpotenzial betroffener Stakeholder von Bedeutung. Die strategische Bewertung erfolgt mit Blick auf die Frage, inwieweit die Berücksichtigung der Stakeholder zur Erfüllung langfristiger Ziele beiträgt.

Hinsichtlich der Auswahl von relevanten Stakeholdern erscheint ein zwei-stufiges Vorgehen sinnvoll, welches eine ethisch-normative Bewertung mit einer strategischen Bewertung zusammenführt (Waxenberger 2001, S. 46ff.). So geht es in einem ersten Schritt darum, jene Personengruppen zu identifizieren, deren grundlegenden Rechte von den Unternehmensaktivitäten in besonderer Art und Weise betroffen sind (Legitimität und Betroffenheit als wesentliche Kriterien). In einem zweiten Schritt können zusätzlich solche Stakeholder bzw. deren Anliegen berücksichtigt werden, welche für das Unternehmen aufgrund ihres Einflusses oder aus anderen Überlegungen heraus von besonderem Interesse sind.

Auch bei den identifizierten Issues sind jene herauszufiltern, auf welche die Ressourcen der Organisation am ehesten gelenkt werden sollten. Als Auswahlkriterien werden dabei häufig die Dringlichkeit bzw. die Wichtigkeit der dahinter stehenden Stakeholder, die Auswirkung auf die Organisation und Beeinflussbar- keit durch die Organisation genannt (Liebl 2000, S. 106ff., vgl. auch Tab. 2).

Da Issues in der Regel auf Stakeholder zurückgeführt werden können, ist es sinnvoll, Stakeholderbewertung und Issue-Bewertung zusammenzuführen. Mittels einer Matrix kann sichtbar gemacht werden, welche Themen und Issues mit welchen Stakeholdern verbunden sind und kann die Beurteilung von Dringlichkeit, Legitimität und Auswirkung von Anliegen und Issues mit der Beurteilung des Macht- und Einflusspotenzials von Stakeholdern verknüpft werden. Für die Ableitung von strategischen Prioritäten ist schließlich noch eine Zusammenführung mit den Ressourcen und Kompetenzen des Unternehmens sinnvoll, das heißt die Frage danach, welchen Mehrwert die Organisation mit der Verfolgung eines Themas schaffen kann (vgl. Abb. 16).

\subsubsection{Konsequenzen der Analyse}

Die Analyse von Stakeholderinteressen und Issues sowie der internen Ressourcen und Werte führt zur Aufdeckung gesellschaftlich relevanter Themen, für welche das Unternehmen aus strategischen oder ethischen Überlegungen heraus Verantwortung tragen sollte und bei denen es aufgrund seiner Ressourcen und Fähigkeiten be-

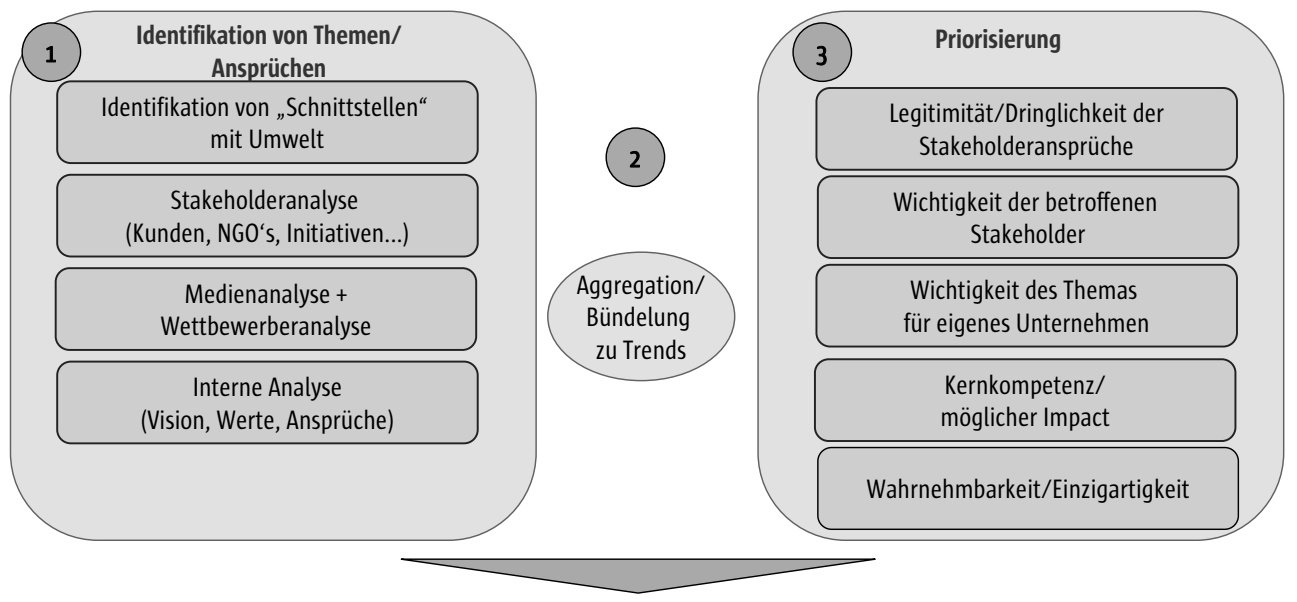

- Definition von Handlungsschwerpunkten

- Formulierung von Zielen und Strategien

- Integration in strategische Programme und Ableitung von Maßnahmen

Abb. 16 Stakeholderdialog und Umfeldanalyse zur Identifizierung und Priorisierung interner und externer Themen 
Tab. 2 Bewertung von Stakeholderanliegen zur Auswahl von Handlungsschwerpunkten

\begin{tabular}{|c|c|c|c|c|}
\hline Kriterien & $\begin{array}{l}\text { Gewicht: } \\
100 \%\end{array}$ & $\begin{array}{l}\text { Thema } 1 \\
\text { (Punkte) }\end{array}$ & $\begin{array}{l}\text { Thema } 2 \\
\text { (Punkte) }\end{array}$ & $\begin{array}{l}\text { Thema x } \\
\text { (Punkte) }\end{array}$ \\
\hline 1. Dringlichkeit/Legitimität der Stakeholderanliegen & $\mathrm{x} \%$ & & & \\
\hline 2. Wichtigkeit der betroffenen Stakeholder & $\mathrm{x} \%$ & & & \\
\hline 3. Wichtigkeit für eigenes Unternehmen & $\mathrm{x} \%$ & & & \\
\hline 4. Kernkompetenz/möglicher Impact & $\mathrm{x} \%$ & & & \\
\hline 5. Wahrnehmbarkeit/Einzigartigkeit & $\mathrm{x} \%$ & & & \\
\hline 6. .... & $\mathrm{x} \%$ & & & \\
\hline Summe & & & & \\
\hline
\end{tabular}

sonderen Mehrwert liefern kann. Sie liefert außerdem Informationen über das im Unternehmen existierende Wertgerüst, welches als Maßstab der Bewertung von Stakeholderanliegen dient und/oder durch das Verfassen von Leitlinien und Grundsätzen manifestiert und gefestigt bzw. gesteuert werden kann.

Wesentlich für die Ableitung strategischer Konsequenzen, welche sich beispielsweise auf Inhalte oder Adressaten der Verantwortung beziehen, ist die Zusammenführung der Ergebnisse von externer und interner Analyse, um extern herangetragene Ansprüche mit Erkenntnissen über den eigenen strategischen Spielraum sowie eigenen Werten und Leitlinien vereinen zu können. In der Regel führt die Analyse aber auch zur Identifizierung von Konflikten zwischen Anliegen unterschiedlicher Stakeholder, für welche sich das Unternehmen gleichermaßen verantwortlich fühlt. Sofern es nicht gelingt, über geeignete Strategien die Interessenkonflikte auszugleichen und zu einer für alle Beteiligten gewinnbringenden Situation zu gelangen, wird eine Interessenabwägung notwendig, das heißt es sind Regeln zu etablieren, nach denen die Priorität unterschiedlicher Ansprüche beurteilt werden können. Diese Regeln, welche z.B. auf die Dringlichkeit von Gütern, die Wahrscheinlichkeit negativer Konsequenzen, die Dauer der Beeinträchtigung, die Anzahl der beeinträchtigten Personen und die Reversibilität der Folgen abzielen (Korff 1979, S. 68ff.), können im normativen Wertegerüst festgeschrieben werden.

\subsection{Die Festlegung der Ziele, Strategien und des strategischen Programms des verantwortlichen Managements}

Auf Basis der Analyse und abgeleitet aus den übergeordneten Zielen des Unternehmens können nun Ziele und Strategien des Managements der sozialen Verantwortung konkretisiert werden. Dabei geht es um die Frage wie die im Rahmen der externen Analyse identifizierten Themen und Ansprüche unter Berücksichtigung eigener Ressourcen und Werte in den langfristigen Handlungsplan des Unternehmens integriert werden können ${ }^{27}$. Resultat ist die Ableitung von Handlungsschwerpunkten sowie die Definition strategischer Ziele und der Wege der Zielerreichung. Zur Auswahl von Handlungsschwerpunkten können die in Kapitel 3.3 definierten Kriterien sowie die grundlegenden Ziele des Managements der Verantwortung in Form eines Scoring Modells oder einer Entscheidungsmatrix berücksichtigt werden (s. Tab. 2).

So definiert z.B. Henkel insgesamt 5 Themenfelder als Schwerpunkte seiner Strategie des Verantwortungsmanagements (vgl. Abb. 17).

Die im Zuge des Verantwortungsmanagements definierten Handlungs- und Themenschwerpunkte sind verknüpft mit dem Zielbildungsprozess, innerhalb dessen formuliert wird, welche Ziele in welchem Ausmaß wie, wann, wo und durch wen erreicht werden sollen

27 Zum Wesen der Strategie (vgl. Schreyögg; Koch 2006, S. 61) mit Bezug auf Verantwortungsmanagement (Münstermann 2007, S. 78-79) 


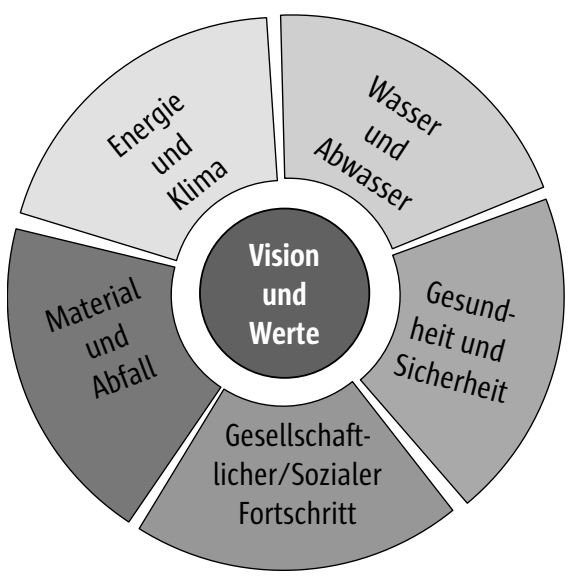

Abb. 17 Fokusfelder bei Henkel (Henkel 2010)

(vgl. Abb. 18, erstellt nach Angaben aus Heinen 1976, S. 59 ff., Münstermann 2007, S. 62-63).

Auch die gewählten Strategien sollten sich an den identifizierten Handlungsschwerpunkten orientieren und Wege zur Erreichung der definierten Ziele darstellen. Dabei sind Ziele und Strategien häufig nicht getrennt formulierbar. Je konkreter die Ziele des Verantwortungsmanagement definiert sind, desto eher werden sie bereits mit Handlungsplänen und Maßnahmen verknüpft; häufig sind Ziele auch erst der Ausfluss bzw. die Konsequenz der Strategiebestimmung (Meffert 2000, S. 69). Die Gestaltung der Strategie bezieht sich dabei auf drei Ebenen, innerhalb derer Aspekte des Verantwortungsmanagement berück- sichtigt werden können (Göbel 2006, S. 148-150): die Unternehmensstrategie, die Geschäftsfeldstrategie und das strategische Programm.

\section{Die Unternehmensstrategie}

Sie befasst sich mit der Reichweite des Geschäfts (das heißt die Wahl der Geschäftsfelder auf nationaler und internationaler Ebene), den Grad der vertikalen Integration des Unternehmens und der Allokation der knappen Ressourcen auf die einzelnen Geschäftsfelder (Schreyögg; Koch 2007, S. 73$)^{28}$. Die Integration von Aspekten der gesellschaftlichen Verantwortung in die Unternehmensstrategie könnte beinhalten, selbst in neue nachhaltige Geschäftsfelder einzutreten (z.B. Social Banking bei Banken, umweltfreundliche Kleinwagen bei Automobilkonzernen oder Medizinische Versorgungszentren bei Krankenhäusern) oder Kooperationen mit entsprechenden Partnern einzugehen. Bei der Allokation der Ressourcen sollten Aspekte der Verantwortung und die Beurteilung der Geschäftsfelder mit eingehen (z.B. tritt Henkel nur dann mit neuen Produktangeboten auf den Markt, wenn diese einen Beitrag in mindestens einem der durch Henkel definierten Fokusfelder der Nachhaltigkeit leistet).

28 Fragen der organisatorischen Gestaltung sowie der Rechtsform und Eigentumsverhältnisse werden in der Regel auch zu den strategischen Entscheidungen auf Gesamtunternehmensebene gezählt, werden aber hier an anderer Stelle behandelt.

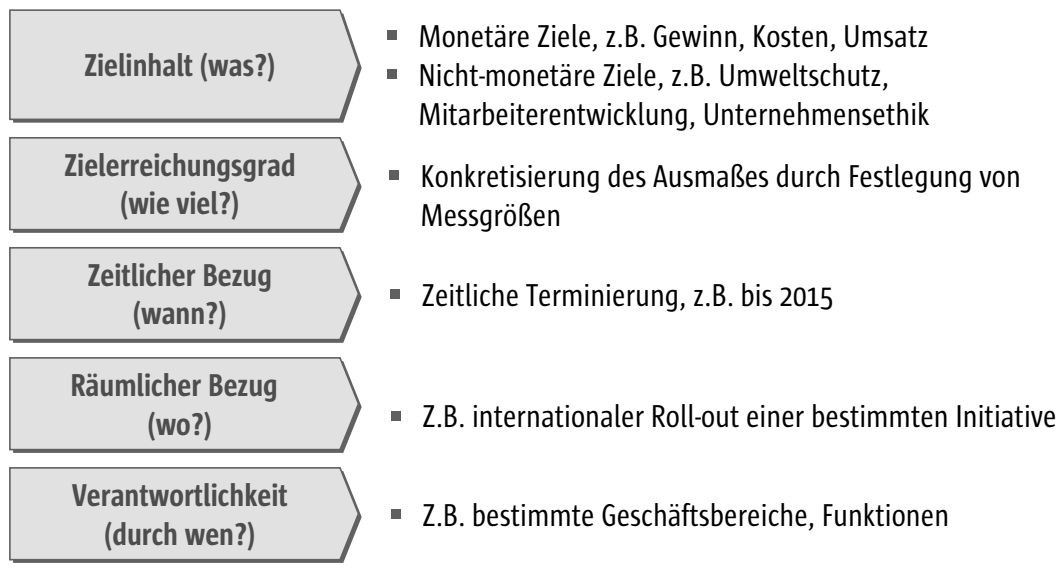

Abb. 18 Inhalte der Zielformulierung 


\section{Die Geschäftsfeldstrategie}

Sie bestimmt den Wettbewerbsvorteil, mit welchem das Unternehmen sich gegenüber dem Wettbewerb positionieren möchte (Porter, 1980). Eine Positionierung im Wettbewerb unter Integration des Verantwortungsgedankens kann sich auf unterschiedliche Elemente der Wertschöpfungskette beziehen, so zum Beispiel auf die Gestaltung des Leistungsangebotes, den Vertrieb, Prozesse oder den Einkauf:

- Gestaltung des Produkt- bzw. Leistungsangebotes (z.B. Gesundheitsversorgung in strukturschwachen Regionen)

- Vertrieb/Marketing: Berücksichtigung ethischer Aspekte bei der Vermarktung von Produkten

- Prozesse: Verantwortlicher Ressourcenverbrauch, verantwortliche Mitarbeiterführung

- Einkauf: Berücksichtigung des ökologischen oder sozialen „footprints“ der für den Betrieb notwendigen Ressourcen

\section{Das strategische Programm}

Als Bindeglied zu den operativen Plänen dient es dazu, die grundlegenden Strategien des Gesamtunternehmens und der Geschäftsfelder in Funktionalstrategien oder im Rahmen von strategischen Projekten umzusetzen.

Die gewählten Strategien sollten sich an den identifizierten Handlungsschwerpunkten orientieren und Wege zur Erreichung der definierten Ziele darstellen.

\subsection{Die Steuerung und Kontrolle}

\subsection{1 Überblick über Steuerungskonzepte}

Steuerungskonzepte bzw. -systeme unterstützen die Umsetzung von Strategien im Untenehmen $^{29}$. Wesentlich hierfür sind die Formulierung von Zielen, Messgrößen und Maßnahmen sowie die regelmäßige Kontrolle der Zielerreichung. Die meisten Steuerungssysteme konzentrieren sich bisher auf den ökonomischen und ökologischen

29 Im Gegensatz zu den Instrumenten bedienen sich Konzepte und Systeme eines Sets systematisch aufeinander abgestimmter Instrumente (z.B. Audit, Leitbild, Bericht usw.) zur Erreichung eines bestimmten Zielbündels.
Bereich (Schaltegger u.a. 2002, S. 11). Steuerungskonzepte, welche das Verantwortungsprinzip auch im sozialen Bereich explizit mit berücksichtigen, sind z.B. die Sustainable Balanced Score Card und der Sustainable Excellence Ansatz ${ }^{30}$. Während der Sustainable Excellence Ansatz bisher im Wesentlichen auf Gesamtunternehmensebene als Selbstbewertungs- bzw. BenchmarkInstrument verwandt wird, dient die Sustainable Balanced Score Card der ganzheitlichen und durchgängigen Steuerung verantwortungsvoller Strategien im Unternehmen (vgl. Kap. 3.5.2 und $3 \cdot 5 \cdot 3)$.

Die Kontrolle ist in der Regel in den internen Steuerungszyklus integriert. Neben einer regelmäßigen Überprüfung der Zielerreichung innerhalb des regulären Controllingzyklus (z.B. Kontrolle von Ressourcenverbrauch, Weiterbildungsscontrolling $u$.ä.) finden außerdem interne oder externe Audits (zum Beispiel Sozialaudit- oder Nachhaltigkeitsaudits, Legal-Compliance-Audit) ${ }^{31}$ oder extern durchgeführte Ratings oder Zertifizierungen Anwendung, bei welchen die Umsetzung des Verantwortungsgedankens bzw. die Einhaltung selbstgesetzter Normen oder Leitlinien bzw. überprüft werden (s. hierzu ausführlich Schaltegger 2007). So ist auch der Sustainable Excellence Ansatz in der Regel mit einem Audit verbunden, innerhalb dessen ausgebildete $\mathrm{Au}$ ditoren leitfragengestützt das Management bewerten. Diese Kontrollinstrumente werden häufig für bestimmte Teilbereiche verwandt, so z.B. zur Überprüfung/Bewertung von Gleichberechtigung, Arbeitssicherheit, Führungsstil/Betriebsklima oder Aus- und Weiterbildung (Schaltegger 2007, S. 16).

Organisatorisch wird die Kontrolle außerdem über die Bildung von Komitees oder die Benennung von Verantwortlichen bestimmt, welche als Anlaufstelle bei ethischen Fragestellungen dienen und Entscheidungen von Führungskräften vor dem Hintergrund ethischer Fragestellungen überprüfen (z.B. Ethikomitees).

30 Einen weiteren wichtigen Ansatz stellt das Sustainable Value Management dar, auf welches im Folgenden nicht weiter eingegangen wird (vgl. Steimle 2008, S. 116).

31 Der SA 8000 ist beispielsweise ein Standard zur Erfassung und Verbesserung sozialer Leistungen von Unternehmen. Er ist aber insbesondere für global tätige Unternehmen als "Mindeststandard“ relevant. 


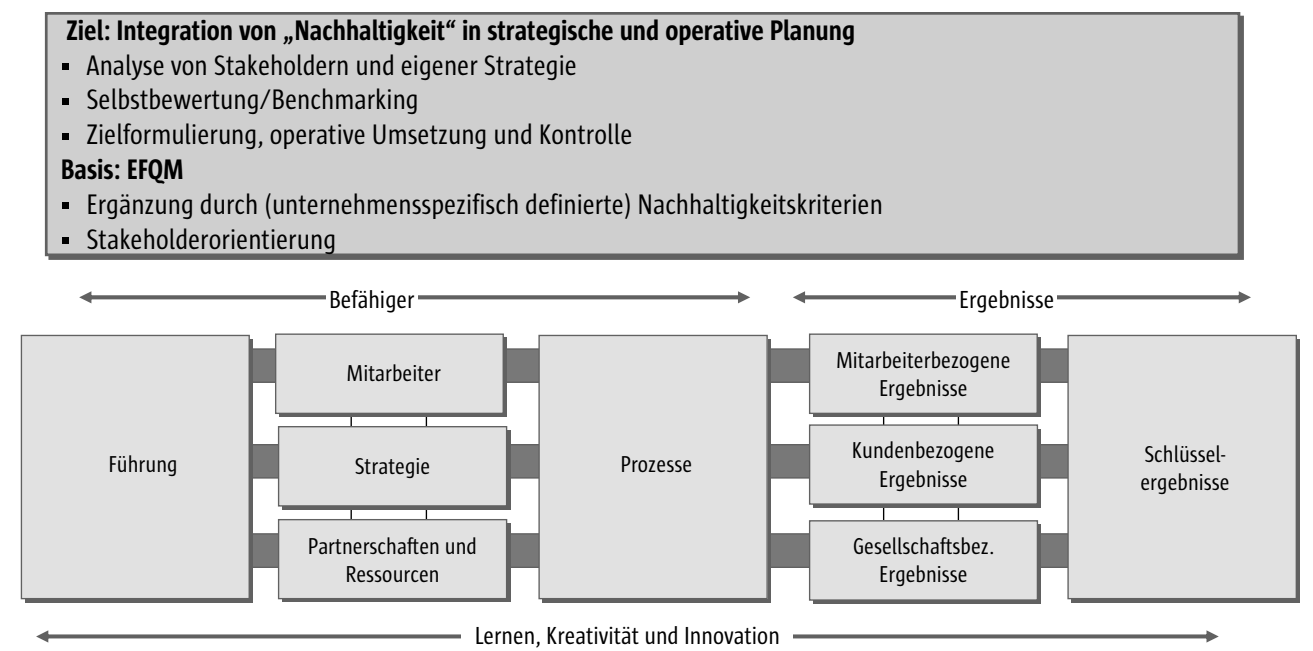

Abb. 19 Das EFQM-Modell als Basis des Sustainable Excellence-Ansatzes (in Anlehnung an @ European Foundation for Quality Management 2010, mit freundlicher Genehmigung)

\subsubsection{Der Sustainable Excellence Ansatz}

Der Sustainable Excellence Ansatz stellt eine Weiterführung des Excellence Ansatzes der EFQM dar (s. Abb. 19), welcher auf den Prinzipien des Total Quality Managements beruht. Durch eine Bewertung des Ressourcenpotenzials (insbesondere Führung, Mitarbeiter, Partnerschaften u.ä.), der Unternehmensprozesse und der Unternehmensergebnisse sollen Ansatzpunkte für eine Verbesserung der nachhaltigen Unternehmensperformance erreicht werden. Dabei wird das Prinzip der Verantwortung zum integralen Bestandteil der strategischen und operativen Führung des Unternehmens (Rabe v. Pappenheim 2008, S. 107).

Die Bewertung erfolgt über verschiedene, angepasste oder standardisierte Erhebungs- und Bewertungsbögen. Dabei wird die sogenannte RADAR-Methodik angewandt, welche ausgehend von den gewünschten Zielen das Vorgehen und die Umsetzung definiert und in der Bewertung überprüft, inwieweit die Ergebnisse gemessen werden und daraus Verbesserungspotenziale abgeleitet werden.

Der Sustainable Excellence Ansatz wird seit 2006 von einigen Unternehmen des Mittelstandes erprobt, allerdings steht eine endgültige Überarbeitung des Modells durch die EFQM noch aus.

\subsubsection{Die Sustainable Balanced Score Card}

Auch die Sustainable Balanced Score Card ist eine Erweiterung eines Controlling-Instrumentes, welches zur Planung, Steuerung und Kontrolle ökonomischer Zielsetzungen entwickelt wurde, um Aspekte der sozialen und ökologischen Verantwortung (Figge et al. 2001, S. 2). In seiner Grundform dient die Balanced Score Card der ausgewogenen Steuerung von vier Bereichen bzw. Perspektiven, welche den langfristigen Erfolg eines Unternehmens sowie dessen Strategie und Vision maßgeblich beeinflussen, und definiert für diese Bereiche strategische Ziele, Ergebniskenngrößen, Leistungstreiber sowie Maßnahmen für die Erreichung der Ziele (Kaplan; Norton 1992, 1997, 2001).

Grundannahme der Balanced Scorecard ist, dass nicht nur die effiziente Nutzung des Anlagekapitals maßgeblich für den Unternehmenserfolg ist, sondern auch weiche Faktoren wie das intellektuelle Kapital wie das Wissen der Mitarbeiter, effektive Prozesse und gute Kundenbeziehungen. Dementsprechend gehören zu den Perspektiven neben der Finanzperspektive die Kundenperspektive, die Prozessperspektive und die Lern- und Entwicklungsperspektive. Die Perspektiven stehen in einer hierarchischen Beziehung zueinander: Finanzielle Ergebnisgrößen werden maßgeblich von der Kundentreue beeinflusst, diese wiederum ist ein Resultat effizienter Prozesse, wel- 
Tab. 3 Generische strategische Kernaspekte für die Formulierung von Ergebniskennzahlen

\begin{tabular}{|c|c|c|c|}
\hline Finanzperspektive & Kundenperspektive & Prozessperspektive & $\begin{array}{l}\text { Lern- und } \\
\text { Entwicklungsperspektive }\end{array}$ \\
\hline $\begin{array}{l}\text { Optimierung der Erträge } \\
\text { und Abbau Defizit } \\
\text { Erschließung neuer } \\
\text { Finanzquellen } \\
\text { Steigerung der } \\
\text { Investitionsquote }\end{array}$ & $\begin{array}{l}\text { Patientenzufriedenheit } \\
\text { Zufriedenheit der } \\
\text { einweisenden Ärzte } \\
\text { Reputation/ } \\
\text { Bekanntheitsgrad } \\
\text { Wissenschaftliche und } \\
\text { klinische Kooperationen }\end{array}$ & $\begin{array}{l}\text { Innovationsprozess Schaffung } \\
\text { neuer Leistungsangebote } \\
\text { Betriebsprozess } \\
\text { Verbesserung der internen } \\
\text { und externen Kommunikation } \\
\text { Harmonisierung der Prozesse } \\
\text { - Schaffung von Transparenz } \\
\text { Kundendienstprozess } \\
\text { Verbesserung von Patienten- } \\
\text { aufnahme/Information }\end{array}$ & $\begin{array}{l}\text { Steigerung der Mitarbeiter- } \\
\text { zufriedenheit und der } \\
\text { Gleichberechtigung } \\
\text { Ausbau der Qualifikation auf } \\
\text { allen Ebenen } \\
\text { Ausbau der Informationssysteme }\end{array}$ \\
\hline
\end{tabular}

che durch kompetente und motivierte Mitarbeiter gestaltet werden. Im Krankenhausmanagement könnten für die einzelnen Perspektiven beispielsweise die in Tabelle 3 dargestellten strategischen Ziele definiert werden (erstellt nach den Angaben aus Conrad 2001, S. 81-92).

Die Patientenzufriedenheit könnte zum Beispiel über Patientenbefragungen gemessen werden und ein entsprechender Zufriedenheitsindex als Ergebniskennzahl definiert werden. Leistungstreiber wäre z.B. die schnelle Versorgung, ausreichende Information, die wiederum entsprechende Maßnahmen nach sich ziehen würden.

Schon in der klassischen Form der Balanced Scorecard werden die Belange von Kunden (Patienten) und Mitarbeitern als wesentliche Stakeholder über die Kunden-, Mitarbeiter- und Prozessperspektive mit angesprochen. Bei einer Sustainable Balanced Scorecard kann der Verantwortungsgedanke zusätzlich berücksichtigt werden über

- die Definition von entsprechenden sozialen und umweltbezogenen Kennzahlen innerhalb der bestehenden Perspektiven,

- die Etablierung einer neuen Perspektive oder

- die Erstellung einer eigenen Sustainable Balanced Scorecard (Figge et al. 2001, S. 22).

Bei einer Integration entsprechender Kennzahlen in die bestehenden Perspektiven könnten z.B. ökologische Aspekte bei betrieblichen Prozessen oder Produktgestaltung oder die Zufriedenheit zusätzlicher Stakeholder in der Kundenperspektive berücksichtigt werden. Dies ist dann sinnvoll, wenn die entsprechenden Größen positiv auf finanzielle Ziele wirken und quasi in den Markt- mechanismus integriert sind (z.B. Anbieter von Ökowaschmittel). Durch die Etablierung einer zusätzlichen Nicht-Marktperspektive wird gewährleistet, dass auch die Aspekte der Verantwortung berücksichtigt werden, die nicht in den Marktmechanismus integriert sind und dennoch Kernaspekte der Strategie darstellen (Figge et al. 20o1, S. 26). Abbildung 20 zeigt auf, wie eine Sustainable Balanced Scorecard mit zusätzlicher Perspektive gestaltet werden könnte.

\subsection{Die Kommunikation}

Kommunikation wird häufig als „Austausch“ oder „Übertragung “ von Informationen beschrieben. Gemäß Mackenzie (Mackenzie 1969) ist die Kommunikation integraler Bestandteil wesentlicher Managementfunktionen, insbes. des Personaleinsatzes, der Führung und der Kontrolle. Gleichzeitig dient sie im Bereich des Marketings der zielgerichteten Beeinflussung interner und externer Zielgruppen (s. Kap. 3.1). Bezogen auf das Management sozialer Verantwortung ist Kommunikation in folgenden Bereichen relevant:

\section{Externe Analyse und Kommunikation}

- Wie in Kapitel 3.3.2 erläutert, ist die Analyse der Stakeholdererwartungen wesentlich für die Definition von Zielen und Maßnahmen des Verantwortungsmanagement; die Kommunikation der Ergebnisse ist dann die logische Konsequenz. Ein permanenter Stakeholderdialog ist dabei eine effektive Möglichkeit, 


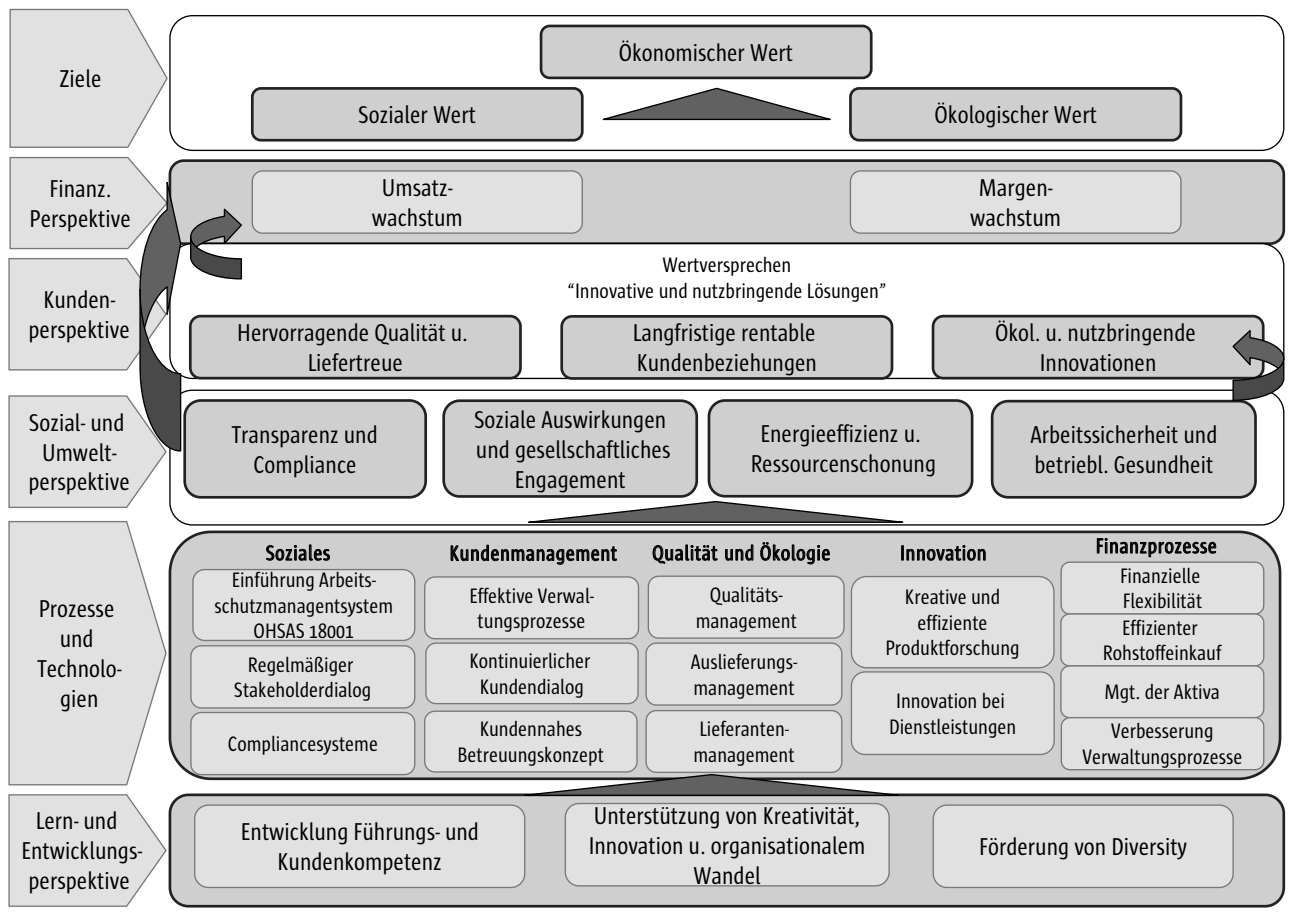

Abb. 20 Exemplarische Sustainable Balanced Scorecard mit zusätzlicher Perspektive für ein Industrieunternehmen

diese Erwartungen zu erkennen, Stakeholdererwartungen miteinander in Einklang zu bringen und relevante Gruppen über die Ergebnisse des Verantwortungsmanagements zu informieren. Der Grundgedanke des Prinzips der Verantwortung - nämlich das Finden eines für alle Beteiligten akzeptablen Ausgleichs zwischen verschiedenen und gegebenenfalls konfligierenden Interessen - stellt die Kommunikation bzw. den Dialog sogar in das Zentrum des Verantwortungsmanagements.

- Fraglich ist, wie ein solcher Stakeholderdialog auch institutionell etabliert werden kann. In Unternehmen der Privatwirtschaft werden allgemeine Fragestellungen häufig im Rahmen von Stakeholderforen diskutiert (s. Kap. 3.3.2). Über Kundenbeiräte werden Kunden in Entscheidungsprozesse involviert, und Mitarbeiter können über Aufsichtsräte und Betriebsrat ihren Einfluss geltend machen. In Krankenhäusern wird hingegen über die Einführung von Ethikkomitees diskutiert. Wesentlich bei der Diskussion um die Institutionalisierung des Stakeholderdialoges ist dabei einerseits die Repräsentativität - das heißt die Integration aller wesentlichen Stakeholdergruppen - und die Verzahnung mit dem strategischen Management - das heißt die Ergebnisse solcher Beiräte oder Foren sollten sich in strategischen Entscheidungen tatsächlich niederschlagen können.

- Schließlich geht es bei der Kommunikation auch um die Vermittlung der Erfolge des Verantwortungsmanagements nach außen. Kommunikation wird daher im Zuge des externen Marketings eingesetzt, um ein positives Bild vom Unternehmen zu schaffen und sich von den Wettbewerbern abgrenzen zu können.

\section{Führung und Koordination}

- Kommunikation dient der Schaffung eines gemeinsamen Verständnisses über Ziele und Maßnahmen des Verantwortungsmanagements bei Mitarbeitern und Führungskräften. Grundsätze und Leitlinien des Verantwortungsmanagements sollten über geeignete Kommunikationskanäle vermittelt und dessen Verankerung über Training und Work- 
shops unterstützt werden. Die Integration von Zielen, Maßnahmen und Ergebnissen in allg. Controllingroutinen (welche sogenannte ,weiche Faktoren " mit berücksichtigen) ist darüber hinaus wesentlich für die Informationsversorgung der Beteiligten (s. Kap. 3.5.3). Dabei geht es nicht nur um die Instrumente selber (z.B. Sustainable Balanced Score Card; Sozial- oder Umweltaudit), sondern insbesondere auch um die Kommunikation der Ergebnisse (nach innen und nach außen).

\subsection{Zusammenfassung}

Die Umsetzung des Verantwortungsprinzips erfordert die Integration des Verantwortungsgedankens in das strategische Management unter Nutzung allgemeiner Managementinstrumente. Auf Basis der grundlegenden Werte und Ziele des Manage- ments müssen Stakeholder und deren Ansprüche analysiert und priorisiert werden und Strategien und Maßnahmen definiert werden, welche zu einem für alle Beteiligten akzeptablen Ergebnis führen werden. Da Stakeholder im Konzept des Verantwortungsmanagements eine wesentliche Rolle einnehmen, müssen sie stärker in den Managementprozess involviert werden, als dies bisher der Fall war. So reicht es nicht aus, sich im Rahmen einer Stakeholderanalyse Klarheit über deren Erwartungen zu verschaffen, vielmehr ist erforderlich, gemeinsam mit Stakeholdern über Konflikte und Konfliktlösungsstrategien nachzudenken und die Ergebnisse des Verantwortungsmanagements regelmäßig auch an diese zu kommunizieren. Im Ergebnis führt dies zu einer höheren Akzeptanz der Entscheidungen der Unternehmensführung bei Mitarbeitern und anderen Stakeholdern und kann so die Differenzierung von möglichen Wettbewerbern zur Folge haben. 